\title{
Cystatin C-Based Renal Function in Predicting the Long-Term Outcomes of Chronic Total Occlusion After Percutaneous Coronary Intervention
}

\section{OPEN ACCESS}

Edited by:

Cristina Vassalle,

Gabriele Monasterio Tuscany

Foundation (CNR), Italy

Reviewed by:

Harry Staines,

Independent Researcher, York, United Kingdom

Ismail Dogu Kilic,

Pamukkale University, Turkey

*Correspondence:

Yue Wu

yue.wu@xjtu.edu.cn

Xiao-Zhen Zhuo

imxiaozhen@xjtu.edu.cn

tThese authors have contributed equally to this work

Specialty section:

This article was submitted to General Cardiovascular Medicine,

a section of the journal

Frontiers in Cardiovascular Medicine

Received: 23 July 2020

Accepted: 29 September 2020

Published: 09 November 2020

Citation:

Li B, Rong J, Wang B, Gao K, Wen X, Li H, Cheng L, Hua Y-M, Li S, Jian Z,

Zhang Y, Huang $H$, Pan $Y, W u Y$ and

Zhuo X-Z (2020) Cystatin C-Based Renal Function in Predicting the Long-Term Outcomes of Chronic Total

Occlusion After Percutaneous Coronary Intervention.

Front. Cardiovasc. Med. 7:586181.

doi: 10.3389/fcvm.2020.586181

\begin{abstract}
Bolin $\mathrm{Li}^{1+}$, Jie Rong ${ }^{2+}$, Bobo Wang ${ }^{3}$, Ke Gao ${ }^{1}$, Xing Wen ${ }^{4}$, Hongbing $\mathrm{Li}^{1}$, Lele Cheng ${ }^{1}$, Yi-Ming Hua ${ }^{1}$, Shanshan Li $^{1}$, Zhijie Jian ${ }^{1}$, Yujing Zhang ${ }^{5}$, Hui Huang ${ }^{5}$, Youlong Pan ${ }^{6}$, Yue Wu ${ }^{1 *}$ and Xiao-Zhen Zhuo ${ }^{\text {** }}$

${ }^{1}$ Department of Cardiology, The First Affiliated Hospital of Xi'an Jiaotong University, Xi'an, China, ${ }^{2}$ Department of Encephalopathy, Affiliated Hospital of Shaanxi University of Chinese Medicine, Xianyang, China, ${ }^{3}$ Health Science Center, School of Pharmacy, Xi'an Jiaotong University, Xi'an, China, ${ }^{4}$ The Center of Gastrointestinal and Minimally Invasive Surgery, The Third People's Hospital of Chengdu, Chengdu, China, ${ }^{5}$ Cardiovascular Department, The General Hospital of Ningxia Medical University, Yinchuan, China, ${ }^{6}$ Cardiovascular and Cerebrovascular Diseases Hospital of Qinghai Province, Xining, China
\end{abstract}

Renal function estimated by various biomarkers predicting for adverse cardiovascular events has not been well-identified in received percutaneous coronary intervention (PCl) for chronic total occlusion (CTO), the advanced stages of atherosclerosis. We aim to determine whether the serum cystatin C-based-estimated glomerular filtration rate (eGFR) can have an improved predictive value in patients with CTO lesions undergoing $\mathrm{PCl}$ as compared with multiple creatinine-based estimates of kidney function. Six hundred and seventy-one patients received CTO PCI were retrospectively included in the study. The eGFR was calculated by modification of diet in renal disease equation for Chinese (cMDRD) and Chronic Kidney Disease Epidemiology Collaboration (CKD-EPI) equations at baseline, respectively. Then, the cohort was categorized into three groups according to standard KDIGO kidney stages based on eGFR. The primary endpoint was all-cause mortality, and the secondary endpoint was cardiac death. Strikingly, cystatin C-based eGFR showed a better performance with the greater area being under the receiver operating characteristic $(\mathrm{ROC})$ curve $(0.73$ for all-cause mortality and 0.73 for cardiac death, separately) and a better assessment for survival free from adverse event across renal levels among four eGFR equations. Compared with eGFR calculated by other formulas, serum cystatin C-based eGFR showed the highest prognostic value for both all-cause mortality (adjusted $\mathrm{HR} 3.6,95 \% \mathrm{Cl} 1.6-8.1, P=0.002$ ) and cardiac death (adjusted HR 2.9, 95\% Cl 1.0-8.1, $P=0.028$ ). Moreover, cystatin C-based eGFR significantly improved the risk reclassification of event with a high value of net reclassification improvement and integrated discrimination improvement. This study may prove that cystatin C-based eGFR is a better predictor of both all-cause mortality and cardiac death than other equations in populations with CTO undergoing PCI.

Keywords: cystatin C, creatinine, estimated glomerular filtration rate, chronic total occlusion, all-cause mortality, cardiac death 


\section{INTRODUCTION}

Renal insufficiency has been found to increase the incidence of both cardiovascular diseases and adverse outcome (1). Estimated glomerular filtration rate (eGFR) has been widely used in clinical practice. Current recommendations and guidelines (2) have pointed out that the Chronic Kidney Disease Epidemiology Collaboration (CKD-EPI) creatinine equation can be applied to estimate GFR with better accuracy than the modification of Diet in Renal Disease (MDRD) equation, as the actual GFR in patients with preserved renal function may be underestimated by using $\operatorname{MDRD}(3,4)$.

Recently, a number of endogenous biomarkers, especially cystatin C, have been established and used to estimate GFR. Cystatin $\mathrm{C}$ is a $13-\mathrm{kDa}$ protein and a member of the family of competitive lysosomal cysteine protease inhibitors. It can be freely filtered by the glomerulus. Compared to creatinine-based eGFR, cystatin C depends less on health status, muscle mass, or other demographic characteristics and appears to be more consistent across cohorts. It has been proved to be a more reliable and sensitive marker for estimating GFR in patients with normal creatinine-based eGFR (5) or with mildly renal insufficiency (6). According to the 2012 Kidney Disease: Improving Global Outcomes (KDIGO) guidelines (2), cystatin C-based eGFR may be used to verify the diagnosis of a reduced creatinine-based eGFR and, therefore, recategorize patients into normal group in the absence of albuminuria. Several studies have shown a very close relationship between cystatin $\mathrm{C}$ and cardiovascular events, especially cardiac death and all-cause mortality (6), among different cohorts, such as patients with chronic kidney disease(CKD) (7) and the elderly people (8). These studies have revealed that cystatin C-based eGFR can be an earlier and stronger predictor for predicting adverse cardiovascular outcomes as compared with creatinine-based eGFR.

Renal dysfunction can be estimated by various equations and has been identified as an important predictor of mortality for patients with coronary heart disease after percutaneous coronary intervention (PCI) and surgical revascularization. Chronic coronary total occlusion (CTO) is advanced atherosclerotic lesions and represents a challenging subset of coronary artery disease. However, the value of eGFR in predicting adverse cardiovascular events has not been well-established within the patients with CTO after PCI. This deficiency forms the basis for this study, aiming to determine whether or not the decreased eGFR calculated by cystatin $\mathrm{C}$ can have a higher risk predictive value for assessing risk and risk grading in terms of all-cause mortality and cardiac death in CTO cohort available in our Cardiology Department.

\section{PATIENTS AND METHODS}

\section{Population and Outcomes}

A total of 762 patients with CTO lesions confirmed by coronary angiography in the Cardiology Department of the First Affiliated Hospital of Xi'an JiaoTong University (Xi'an, Shaanxi, China) undergoing PCI between June 2013 and October 2017 were included in the study. The follow-up for all-cause mortality and cardiac death was carried out via telephone contacts between 2018 and 2019. The study was approved by both Research and Ethics Committees of the First Affiliated Hospital of Xi'an JiaoTong University. Recanalization of CTO lesion was performed according to current guidelines $(9,10)$ by the highly experienced CTO operators with contemporary techniques. A coronary CTO lesion was defined as the presence of Thrombolysis in Myocardial Infarction [TIMI] flow grade 0 within an occluded coronary artery segment of an estimated duration of at least 3 months $(11,12)$. Procedural success was defined by complete restoration of antegraded blood flow (TIMI flow grade 3 ) in the occluded segment with $<30 \%$ residual diameter stenosis. The primary endpoint was all-cause mortality, and the secondary endpoint was cardiac death.

\section{Measurements of Related Parameters}

We collected several demographic, clinical, and analytical parameters. Age, gender, height, and weight were recorded, and body mass index (BMI) $\left(\mathrm{kg} / \mathrm{m}^{2}\right)$ was calculated on the basis of weight and height. Blood pressure (BP) was measured three times or more by a nurse while patients were seated after 5 min of rest. Patients either with persistent $\mathrm{BP}>140 / 90 \mathrm{mmHg}$ or those currently taking antihypertensive drugs were considered hypertensive. Presence of type 2 diabetes mellitus (T2DM) was determined by means of a medical history of diabetes, fasting glucose levels $>126 \mathrm{mg} / \mathrm{dL}(7.0 \mathrm{mmol} / \mathrm{L})$, and/or glycated hemoglobin $(\mathrm{HbA} 1 \mathrm{c})>6.5 \%$. Several parameters, including lowdensity lipoprotein-cholesterol (LDL-c), HDL cholesterol (HDLc), and C-reactive protein, were measured. Serum levels of cystatin $\mathrm{C}$ were determined by an automated particle-enhanced immunoturbidimetric method on a Siemens Dade Behring BN II Nephelometer. Renal function was assessed by means of serum levels of creatinine and cystatin $\mathrm{C}$ at baseline and calculated by MDRD for the Chinese equation (13) (eGFRCMDRD) and chronic kidney epidemiology collaboration (CKDEPI) equations (4), respectively (Supplementary Table 1). CKDEPI equations are composed of eGFR from serum creatinine (eGFRcre), eGFR from serum cystatin C (eGFRcys), and eGFR from the equation incorporating both creatinine and cystatin C (eGFRcre-cys).

\section{Categorization of the Cohorts}

The cohort was categorized according to its prognostic category, defined by an existing classification system-the Kidney Disease: Improving Global Outcomes (KDIGO) guidelines (14) — that is, the level of GFR (known as stages). Therefore, patients were stratified according to eGFR (group 1: eGFR $\geq 90 \mathrm{ml} / \mathrm{min} / 1.73$ $\mathrm{m}^{2}$, group 2: $60-89 \mathrm{ml} / \mathrm{min} / 1.73 \mathrm{~m}^{2}$, group $3:<60 \mathrm{ml} / \mathrm{min} / 1.73$ $\mathrm{m}^{2}$ ) as shown in Supplementary Table 2 .

\section{Statistical Analysis}

Continuous variables are presented as the mean \pm standard deviation if normally distributed or median (lower quartile, upper quartile) otherwise, and Shapiro-Wilk was used for normality test. Categorical variables are presented as numbers and percentages. Differences in the parameters among groups were analyzed using analysis of variance (ANOVA) for normally 
A

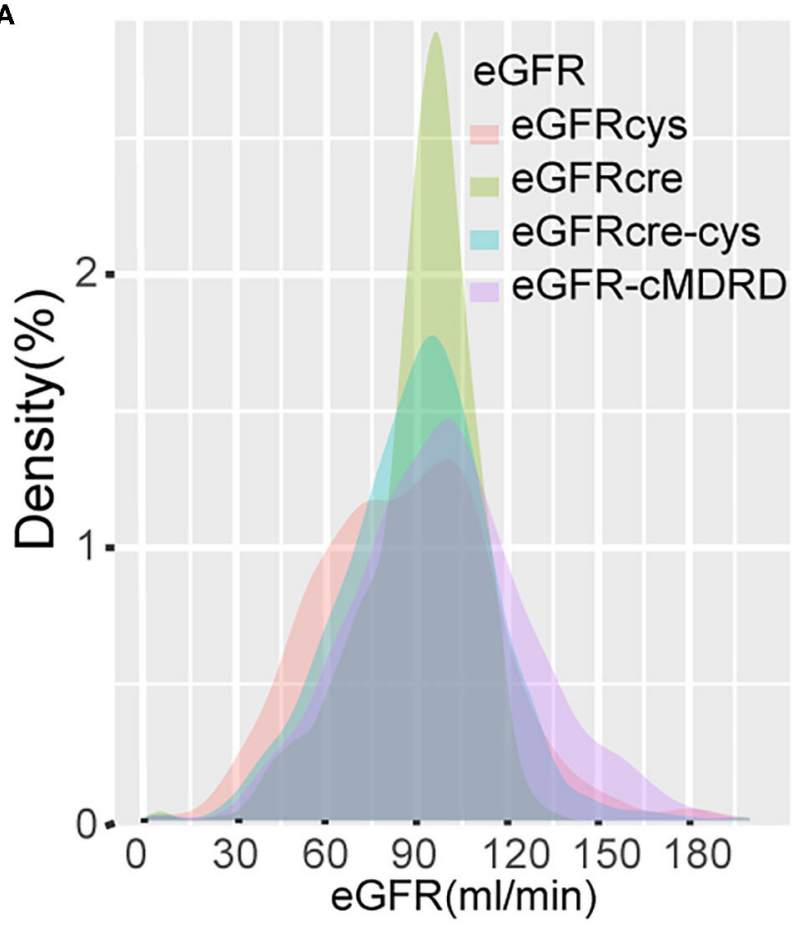

B

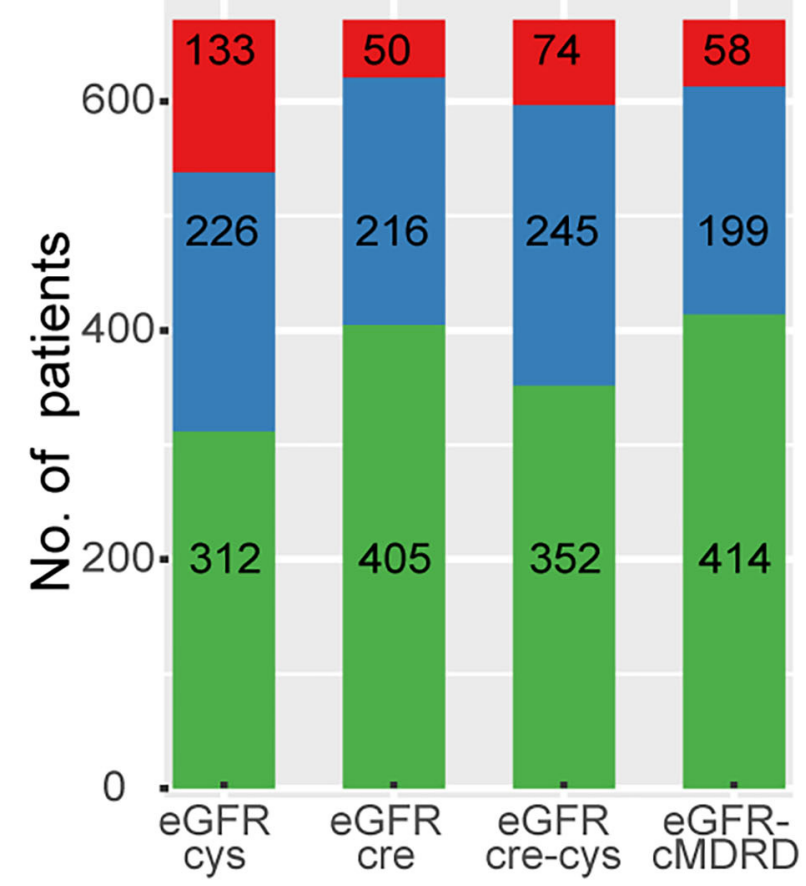

C

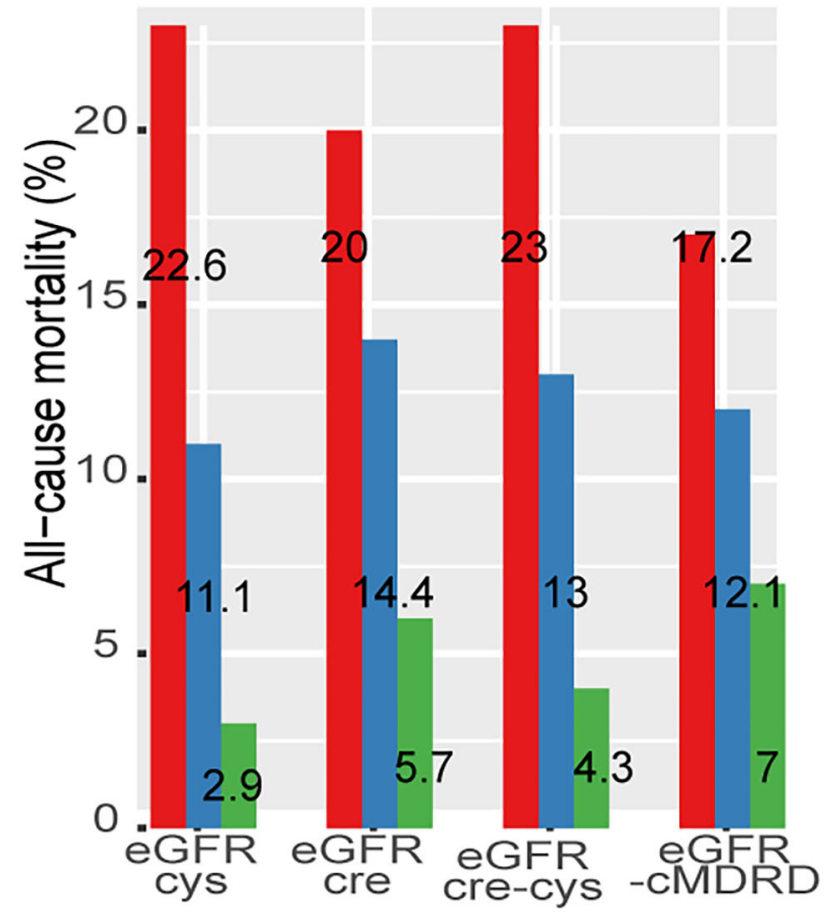

D

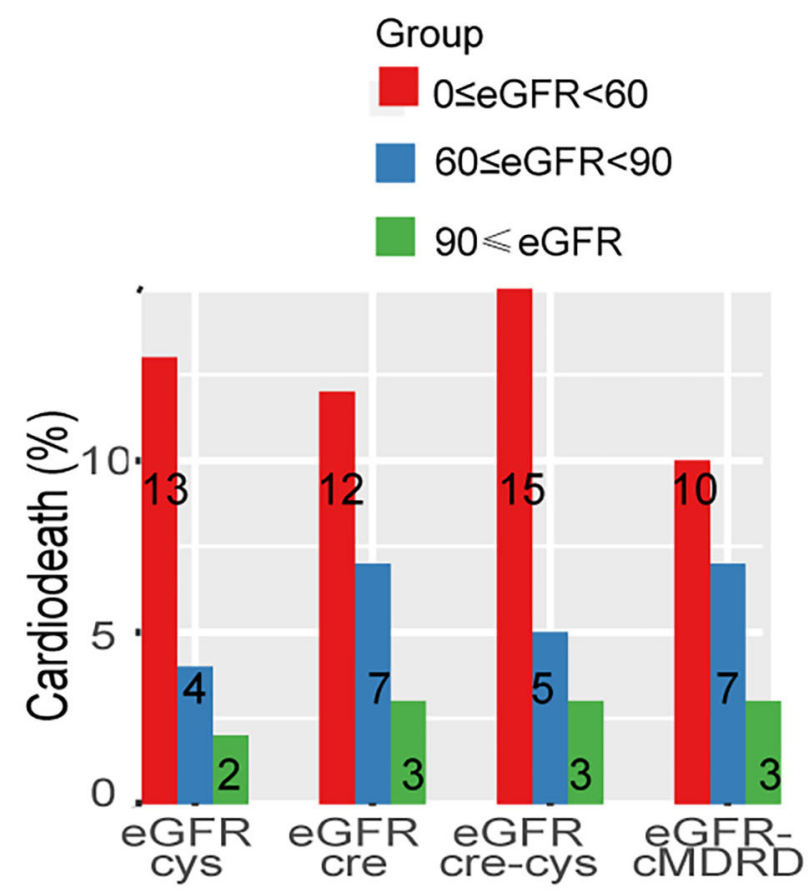

FIGURE 1 | Distribution of eGFR and the rate of adverse event across different renal levels. (A), Distribution of eGFR according to each of the four equations in a density plot. (B), Thresholds of eGFR according to each equation and number of patients in each CKD stage. (C,D), Incidence of all-cause mortality and cardiac death at different renal levels by different formulas. eGFR-cMDRD, calculated by MDRD for Chinese equation. eGFRcre, CKD-EPI equations composed of eGFR from serum creatinine; eGFRcys, eGFR from serum cystatin C; eGFRcre-cys, eGFR from equation incorporating both creatinine and cystatin C. 
TABLE 1 | Baseline characteristics, by GFR, estimated using the eGFRcys equation.

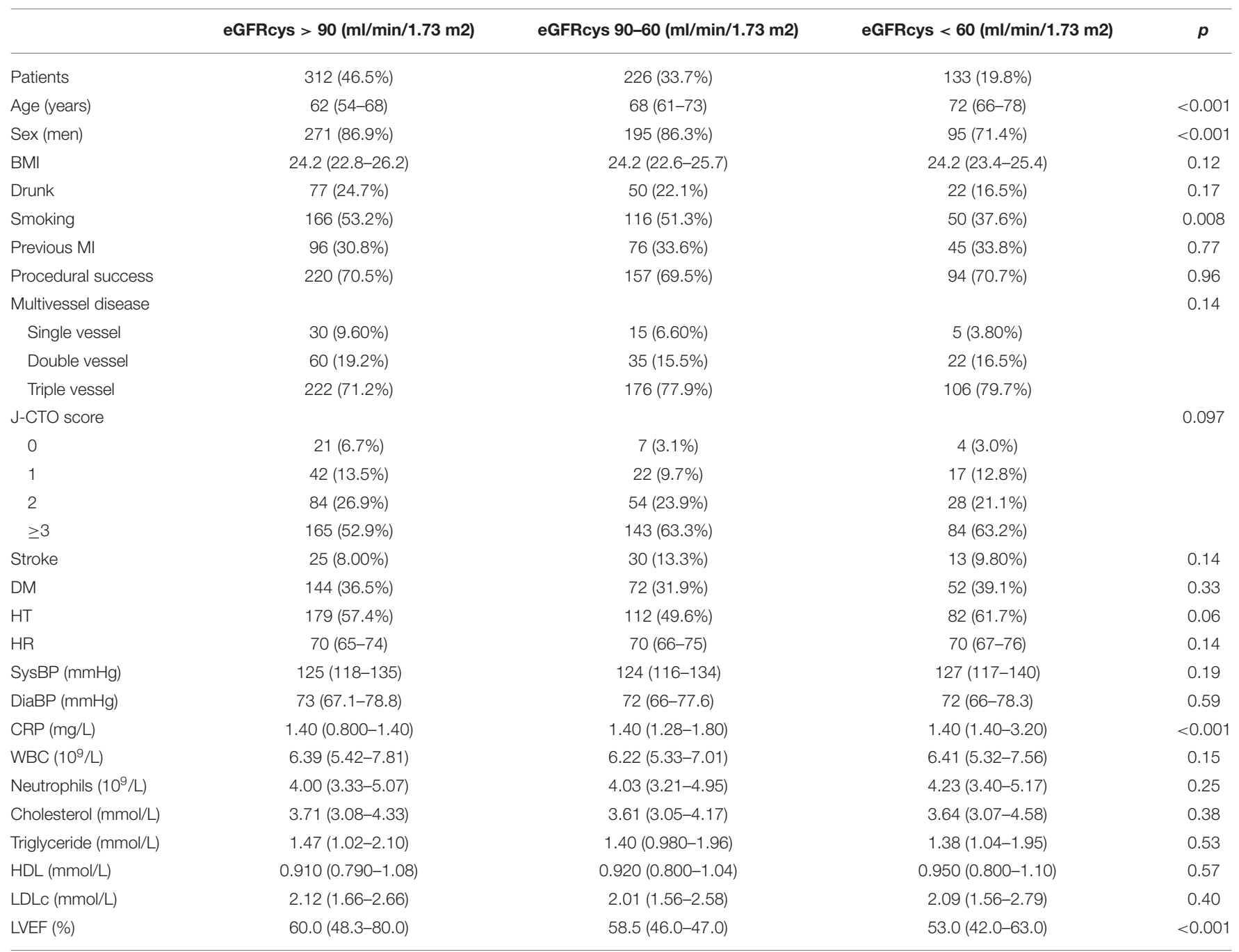

Values are given as median and interquartile range or numbers and percentages.

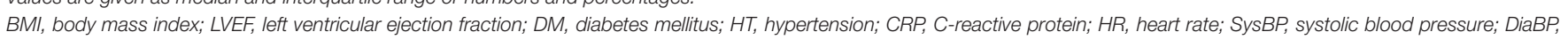
diastolic blood pressure; eGFR, estimated glomerular filtration rate; eGFRcys, eGFR from serum cystatin C.

distributed variables, the Kruskal-Wallis test was used for non-normally distributed continuous variables, and the chisquare test was used for categorical variables. We assessed performance through the receiver operating characteristic (ROC) curve and the area under the ROC curve (AUC). Clinical event rates were compared with the Kaplan-Meier method using the log rank test for comparisons between groups. Cox proportional hazards regression models were used in univariate analyses and multivariate analyses to determine the prognostic value of different kidney stage levels estimated by each eGFR equation. Multivariate analyses adjusted for significant baseline variables and the factors closely related to the outcome of patients with cardiovascular disease, such as age, sex, smoking, BMI, diabetes mellitus (DM), hypertension (HT), and lowdensity lipoprotein cholesterol (LDL-c), left ventricular ejection fraction (LVEF), C-reactive protein (CRP), and procedural success. Net reclassification improvement (NRI) and integrated discrimination improvement (IDI) were performed to analyze the degree to which eGFR improved the predictive ability of the eGFRcys. Both NRI and IDI values were analyzed with their 95\% CI. SPSS version 25.0.0 (IBM, USA) and R version 3.6.1 (https:// www.r-project.org) were used for conducting statistical analyses, and a two-sided $P<0.05$ was considered statistically significant. There is no adjustment for multiplicity.

\section{RESULTS}

A total of 97 patients with acute coronary syndrome at admission were excluded. A total of 762 patients with CTO lesions confirmed by coronary angiography were treated with PCI, and the rate of CTO lesion revascularized was $70.2 \%$. After a median follow-up of 33 months, 91 patients were lost to follow-up, leaving 671 in the study population at the end of follow-up (Supplementary Figure 1). In our cohort, 64 patients (9.5\%) died 

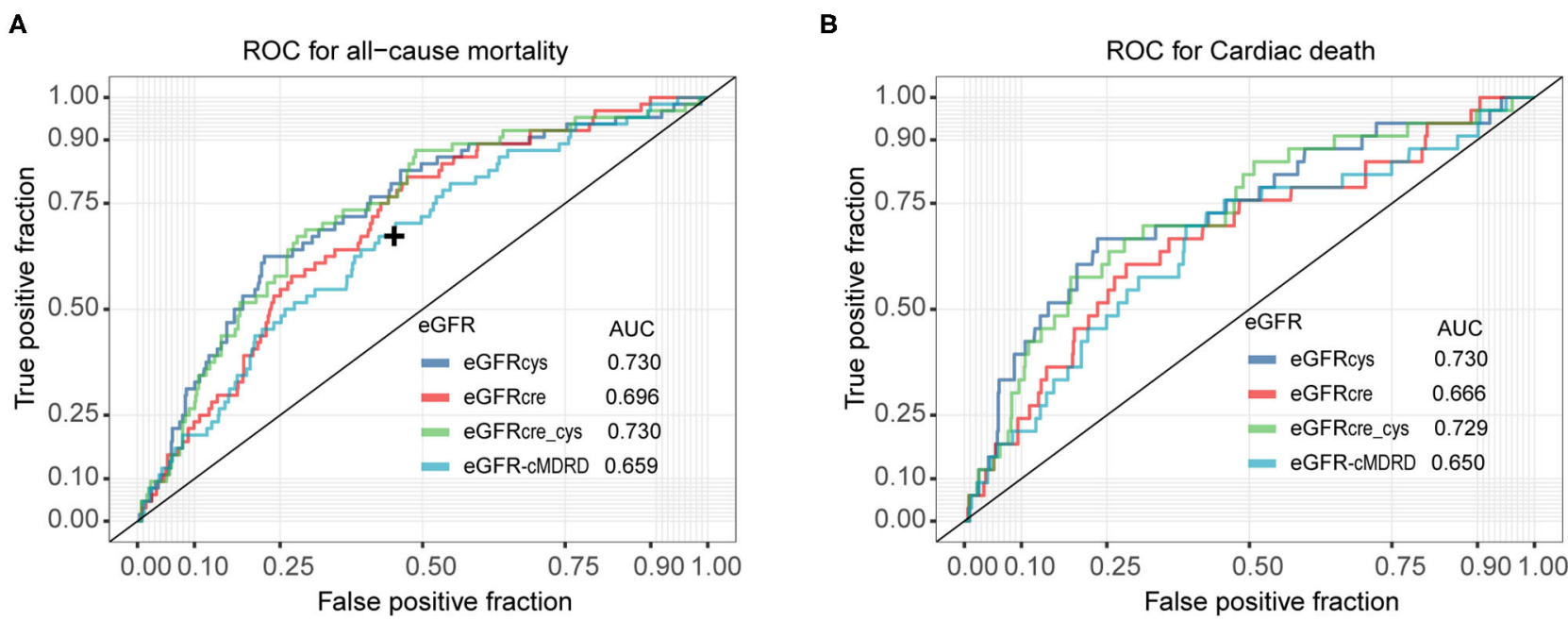

FIGURE 2 | ROC for predicting all-cause mortality and cardiac death. (A), accuracies of eGFRcys, eGFRcre, eGFRcre-cys, and eGFR-cMRDR for predicting all-cause mortality presented as areas under the receiver operating characteristic curves, individually. (B), accuracies of eGFRcys, eGFRcre, eGFRcre-cys, and eGFR-cMRDR for predicting cardiac death presented as areas under the receiver operating characteristic curves, separately. ROC, receiver operating characteristic curve; AUC, area under the receiver operating characteristic curve. DeLong's test to check the difference of AUC for eGFRs calculated by other formulas, compared to eGFRcys. + represents significant differences $(P<0.05)$.

and 33 patients $(4.9 \%)$ died from cardiac death. Distribution of eGFR according to each of the four equations is shown in Figure 1A. Differences in grouping situation of four equations are shown in Figure 1B.

The baseline characteristics of the cohort are shown in Table $\mathbf{1}$. With decrease of renal function, patients were older $(p<0.001)$ with fewer men $(p<0.001)$ and had a higher prevalence of smoking ( $p<0.01)$, a lower (although within normal range) ejection fraction $(p<0.001)$, and increased levels of CRP as well. Procedural success rate, prevalence of DM and HT, serum levels of LDLc, and other baseline parameters did not differ among the three groups.

Levels of renal function estimated by each eGFR equation (eGFR-cMDRD, eGFRcys, eGFRcre, and eGFRcre-cys) were classified according to the risk event of all-cause mortality and cardiac death (Figures 1C,D, Supplementary Table 3). All the groups showed a trend that all-cause mortality was increased with the decreased renal function (eGFRcys: group 1 to group 3, 2.9-22.6\%; eGFRcre: group 1 to group 3 5.7-20.0\%; eGFRcrecys: group 1 to group 3, 4.3-23.0\%; and eGFR-MDRD: group 1 to group $3,7.0-17.2 \%)$. The same was true when cardiac death rates were tested (eGFRcys: group 1 to group 3, 1.90-12.80\%; eGFRcre: group 1 to group 3, 2.70-12.00\%; eGFRcre-cys: group 1 to group 3, 2.60-14.90\%; and eGFR-MDRD: group 1 to group 3, 3.4-10.3\%).

The accuracies of eGFR-cMDRD, eGFRcys, eGFRcre, and eGFRcre-cys for predicting all-cause mortality and cardiac death are shown as the AUC in Figure 2, Supplementary Table 4. For predicting all-cause mortality, the eGFRcys $(\mathrm{AUC}=0.73)$ and eGFRcre-cys $(A U C=0.73)$ exhibited higher predictive accuracy (Figure 2A). For predicting cardiac death, eGFRcys (AUC = 0.73 ) showed the best prediction performance (Figure 2B).
Kaplan-Meier curves were used to illustrate the survival free from adverse events. Overall, patients at a lower eGFR levels had a significantly worse outcome of survival free from all-cause mortality during the follow-up period (Figures 3A-D). eGFRcys showed better performance on grading and risk assessment. Alike, the lower renal function is, the worse survival free from cardiac death is (Figures 3E-H). Also, eGFRcys showed the superior performance on grading and risk assessment as well as eGFRcre-cys to other equations.

We performed Cox regression hazard models on eGFR calculated by each equation as the categorical variable grouped as previously mentioned. The risk of each adverse outcome was assessed by comparing Group 1 (reference) with Groups 2 and 3 in every equation. Cystatin C-based eGFR (adjusted $\mathrm{HR}, 3.6 ; 95 \% \mathrm{CI}, 1.6-8.1 ; P=0.002$ ) was a stronger predictor than eGFRcre (adjusted HR 1.7, 95\% CI 0.7-4.1, $P=0.203$ ), eGFRcre-cys (adjusted HR, 2.3; 95\% CI, 1.0-5.3; $P=0.049$ ), and eGFR-cMDRD (adjusted HR, 1.3; 95\% CI, 0.6-2.9; $P=$ $0.516)$ for the risk of all-cause mortality for moderate-to-severe reduction in GFR (eGFR $\left.<60 \mathrm{ml} / \mathrm{min} / 1.73 \mathrm{~m}^{2}\right)$. Likewise, cystatin C-based eGFR (adjusted HR, 2.9; 95\% CI, 1.0-8.1; $P=0.028)$ was able to predict the risk of cardiovascular mortality better than eGFRcre (adjusted HR, 2.3; 95\% CI, 0.77.6; $P=0.155$ ), eGFRcre-cys (adjusted HR, 2.4; 95\% CI, 0.87.2; $P=0.12$ ), and eGFR-cMDRD (adjusted HR, 1.5; 95\% CI, $0.5-4.2 ; P=0.498)$ for moderate-to-severe reduction in renal function.

The above data showed that in terms of outcome of allcause mortality, eGFRcre was able to estimate an increased risk that was 1.7 times higher than that of the population without CKD (patients whose eGFR was above $90 \mathrm{ml} / \mathrm{min} / 1.73$ $\mathrm{m}^{2}$ ). On the other hand, eGFRcys showed an increased 


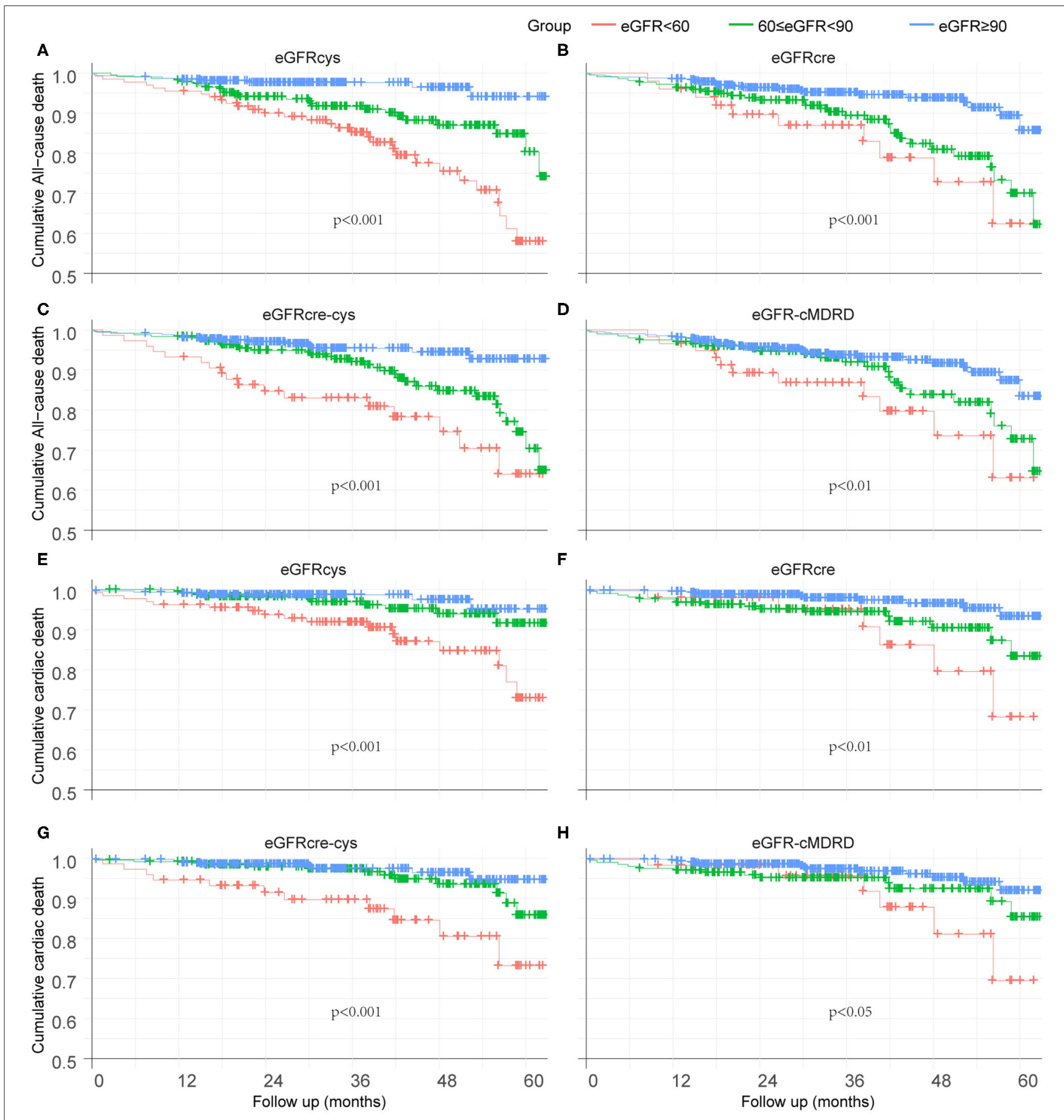

FIGURE 3 | Kaplan-Meier curves to illustrate the survival free from adverse event. (A-D) Survival analysis of eGFRcys, eGFRcre, eGFRcre-cys, and eGFR-cMRDR for all-cause mortality, individually. (E-H) Survival analysis of eGFRcys, eGFRcre, eGFRcre-cys, and eGFR-cMRDR for cardiac death, separately. No. at risk = number of patients at risk for all-cause mortality or cardiac death.

risk rate of 3.6-folds, respectively, as compared with that of the population without CKD. This means that cystatin C is able to improve the predictive value by $111.7 \%$ (for the eGFRcys) in patients with moderate-to-severe reduction in GFR in terms of all-cause mortality. Likewise, cystatin C-based
eGFR may improve the estimation of risk by $26.1 \%$ (for the cystatin C-based equation) in terms of cardiovascular mortality, as compared with those estimated with other equations. A forest plot summarizing these data is shown in Figure 4, Supplementary Tables 5, 6 . 


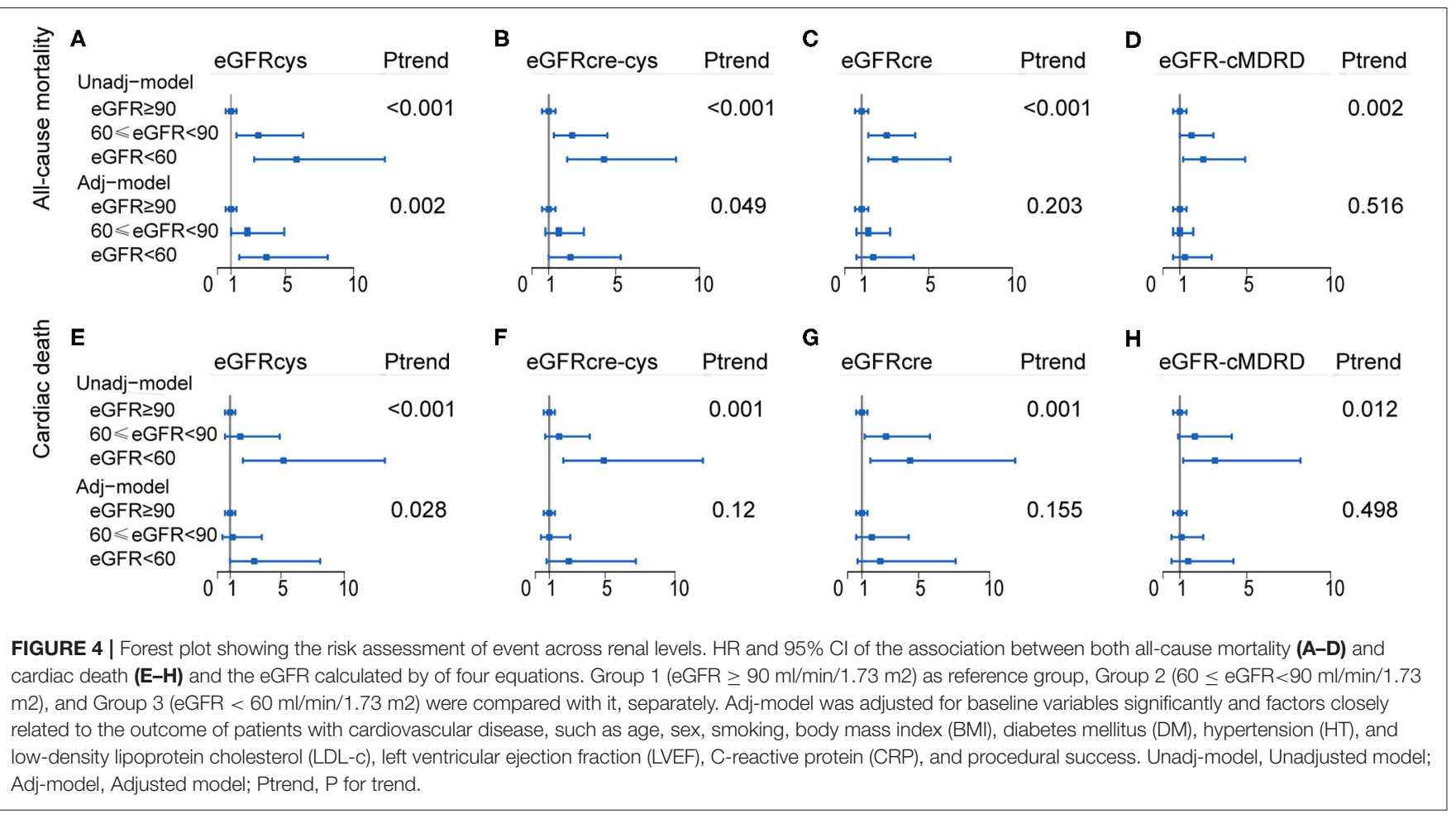

\section{Character}

\section{C-index}

NRI $(95 \% \mathrm{Cl})$

IDI $(95 \% \mathrm{Cl})$

All-cause death

\begin{tabular}{|c|c|c|c|}
\hline eGFRcys & $0.730(0.665-0.795)$ & Reference & Reference \\
\hline eGFRcre & $0.696(0.634-0.757)$ & $-0.498(-0.739--0.258) \longmapsto$ & $-0.025(-0.038--0.013)$ \\
\hline eGFRcre-cys & $0.73(0.667-0.793)$ & $-0.243(-0.498-0.011)$ & $-0.004(-0.010-0.001)$ \\
\hline eGFR-cMDRD & $0.659(0.592-0.726)$ & $-0.492(-0.732--0.252) \longmapsto$ & $-0.029(-0.042--0.017)$ \\
\hline \multicolumn{4}{|l|}{ CardioDeath } \\
\hline eGFRcys & $0.729(0.635-0.823)$ & Reference & Reference \\
\hline eGFRcre & $0.667(0.569-0.765)$ & $-0.569(-0.892--0.247) \longmapsto$ & $-0.019(-0.030--0.008)$ \\
\hline eGFRcre-cys & $0.724(0.633-0.815)$ & $-0.273(-0.62-0.072)$ & $-0.004(-0.009-9 e-04)$ \\
\hline eGFR-cMDRD & $0.652(0.552-0.751)$ & $\begin{array}{r}-0.575(-0.899--0.253) \longmapsto \\
-1\end{array}$ & $-0.023(-0.034--0.011)$ \\
\hline
\end{tabular}

FIGURE $\mathbf{5}$ | Forest plot showing net reclassification improvement of renal function calculated by various eGFR equations. Net reclassification improvement in all-cause mortality and cardiac death was assessed by comparing eGFRcre, eGFRcre-cys, and eGFR-cMRDR with eGFRcys (reference), individually. C-index: Harrell's concordance index. NRI, Net reclassification improvement; IDI, integrated discrimination improvement; Cl, confidence interval.

Regarding discrimination, NRI and IDI in all-cause mortality and cardiac death were assessed by comparing eGFRcre, eGFRcre-cys, and eGFR-cMDRD to eGFRcys (reference). Overall, the reclassification of other equations performed worse both on all-cause mortality and cardiac death (Figure 5). Cystatin C-based eGFR also showed the best performance with the maximum C-index (Figure 5) both in all-cause mortality and cardiac death.

\section{DISCUSSION}

This study showed an increase in all-cause mortality and cardiac death after CTO PCI as baseline renal function declined. Patients with renal insufficiency represent a high-risk patient subset. The values of the four measures showed similar accuracy using the area under the receiver operating characteristic curves. The classification into standard kidney stages showed significant 
differences in the survival curves and Cox regression models for both endpoints.

Given the rapidly aging population in the majority of regions of the world, along with the growing prevalence of chronic disease, chronic kidney disease has become a significant contributor to the increased morbidity and mortality (1). Consistent with the findings made in previous studies for patients suffering from both cardiovascular disease and renal impairment $(15,16)$, we found that patients age and the prevalence of adverse cardiovascular event were increased across stages of renal dysfunction in patients received CTOPCI. Patients with renal impairment more frequently presented with a significantly lower (although within normal range) ejection fraction $(p<0.001)$ and increased levels of CRP. We also found some HR $p$-values which are significant in the univariate analyses become non-significant after adjusting for confounders, and this statistical phenomenon mostly occurred in creatinine-based eGFR. The true causal effect between eGFR values and outcome is affected by confounders (17), defined as variables that are associated with both eGFR values and outcome, but influenced by neither. Differences among the eGFR values with respect to risk relationships probably reflect confounding by non-GFR determinants of the filtration markers. It is well-known that the non-GFR determinants of serum creatinine, including age, sex, BMI, DM, and others can confound the associations between the creatinine-based eGFR and outcomes (18). Non-GFR determinants of cystatin $\mathrm{C}$ also exist, though they are quantifiably smaller than those of creatinine (19).

The main novelty of our research centers on the fact that it was specifically performed in patients with CTO. To the best of our knowledge, this is the first study to address the prognostic usefulness of eGFRcys, by comparing it with eGFRcrea, eGFRcre-cys, and eGFR-cMDRD in patients that received CTO PCI. The analysis of our series of patients suggested that eGFRcys might be a better predictor for cardiovascular events in patients with renal impairment $(<60 \mathrm{ml} / \mathrm{min} / 1.73$ $\mathrm{m}^{2}$ ) when compared with the individuals with normal renal function $\left(>90 \mathrm{ml} / \mathrm{min} / 1.73 \mathrm{~m}^{2}\right.$ ). The main finding in our research is that the eGFRcys performed better than other three eGFR equations in assessing the adverse cardiovascular risk in both cardiac death and all-cause mortality in our CTO population.

Consistent with our findings, Shlipak et al. (7, 20) found that serum levels of cystatin $\mathrm{C}$ were strongly predictive of allcause mortality and cardiovascular disease risk, whereas serum levels of creatinine showed relatively poorer performance in predicting adverse outcomes. Peralta et al. (21) demonstrated that the eGFRcys played a more important role than eGFRcre in identifying the high risk of adverse outcome, both in patients with a moderate to severe renal insufficiency (eGFR $<60 \mathrm{ml} / \mathrm{min} / 1.73 \mathrm{~m}^{2}$ ) and in patients with a mild renal insufficiency $\left(60-89 \mathrm{ml} / \mathrm{min} / 1.73 \mathrm{~m}^{2}\right)$. Recently, Shlipak et al.

(6) demonstrated the increased risk of death for eGFRcys for patients with a mild reduction in renal function (60$89 \mathrm{ml} / \mathrm{min} / 1.73 \mathrm{~m}^{2}$ ). Pedro et al. (22) provided the evidence suggesting that the use of eGFRcys improved the role of eGFR in risk stratification of all-cause mortality for patients with non-ST-segment elevated acute coronary syndrome, as compared with eGFR-MDRD. Helmersson-Karlqvist et al. (23) and Lim et al. (24) found that cystatin C-based eGFR was more closely associated with mortality as compared with both eGFRcre and eGFRcre-cys. Peralta et al. (21) also found the benefits of the eGFRcys in reclassifying mortality risk among adults diagnosed with CKD using the eGFRcre who had the highest risk for complications. Similar clinical applications of cystatin $\mathrm{C}$ in a subset of patients would be its use as a confirmatory test, where cystatin $\mathrm{C}$ would predict risk for all-cause mortality and cardiovascular events among hypertensive patients (25). Thus, above results are consistent with our findings that eGFRcys can improve the risk-grading by identify patients at different levels of risk for adverse outcome whose risk has been estimated with eGFRcre.

\section{STRENGTHS}

One strength of our research was the use of eGFRcys to estimate the risk of cardiac death and all-cause mortality with standard statistical methods, which could be better than the use of the other equations. It should be noted that of more significance to our study was the assessment of a distinct subset of population, i.e., CTO patients. Our results may provide physicians with a support for the need of reconsidering which eGFR equation is the best one for assessment of long-term adverse events in CTO patients.

\section{LIMITATIONS}

The research is a single-center retrospective observational design and is restricted in generalization of the findings. Future multicenter prospective study with a larger number of patients and a longer follow-up is required.

\section{CONCLUSION}

This study has demonstrated that all-cause mortality and cardiac death are increased across stages of renal dysfunction, and the decreased eGFR calculated with cystatin $C$ has a higher risk predictive value to assessing risk and riskgrading in terms of all-cause mortality and cardiac death in our CTO population than eGFR calculated by other three equations.

\section{DATA AVAILABILITY STATEMENT}

The data generated during this study are included in this article. Raw data are available upon reasonable request.

\section{ETHICS STATEMENT}

The studies involving human participants were reviewed and approved by Research and Ethics Committees of the First Affiliated Hospital of Xi'an 
JiaoTong University. The patients/participants provided their written informed consent to participate in this study.

\section{AUTHOR CONTRIBUTIONS}

BL: conceptualization, project administration, writing - original draft, and writing - review and editing. JR: methodology, project administration, data analysis, and writing - review and editing. BW, KG, Y-MH, SL, and ZJ: investigation and data curation. XW: data curation and writing - review. HL: resources and data curation. LC: writing - review and editing. $\mathrm{YZ}$ and $\mathrm{HH}$ : writing - review and editing and data curation. YP: data curation. YW: resources, funding acquisition, and writing - review and editing. X-ZZ: supervision, project administration, and writing - review and editing. All authors contributed to the article and approved the submitted version.

\section{REFERENCES}

1. Jha V, Garcia-Garcia G, Iseki K, Li Z, Naicker S, Plattner B, et al. Chronic kidney disease: global dimension and perspectives. Lancet. (2013) 382:26072. doi: 10.1016/S0140-6736(13)60687-X

2. Stevens PE, Levin A, Kidney Disease: Improving Global Outcomes Chronic Kidney Disease Guideline Development Work Group Members. Evaluation and management of chronic kidney disease: synopsis of the kidney disease: improving global outcomes 2012 clinical practice guideline. Ann Intern Med. (2013) 158:825-30. doi: 10.7326/0003-4819-158-11-20130604000007

3. Stevens LA, Levey AS. Clinical implications of estimating equations for glomerular filtration rate. Ann Intern Med. (2004) 141:959-61. doi: 10.7326/0003-4819-141-12-200412210-00013

4. Levey AS, Stevens LA, Schmid CH, Zhang YL, Castro AF III, Feldman HI, et al. A new equation to estimate glomerular filtration rate. Ann Intern Med. (2009) 150:604-12. doi: 10.7326/0003-4819-150-9-200905050-00006

5. Chinda J, Nakagawa N, Kabara M, Matsuki M, Endo H, Saito T, et al. Impact of decreased estimated glomerular filtration rate on Japanese acute stroke and its subtype. Intern Med. (2012) 51:16616. doi: 10.2169/internalmedicine.51.7185

6. Shlipak MG, Matsushita K, Arnlov J, Inker LA, Katz R, Polkinghorne KR, et al. Cystatin $\mathrm{C}$ versus creatinine in determining risk based on kidney function. $N$ Engl J Med. (2013) 369:932-43. doi: 10.1056/NEJMoa1214234

7. Shlipak MG, Sarnak MJ, Katz R, Fried LF, Seliger SL, Newman AB, et al. Cystatin $\mathrm{C}$ and the risk of death and cardiovascular events among elderly persons. N Engl J Med. (2005) 352:2049-60. doi: 10.1056/NEJMoa043161

8. Menon V, Shlipak MG, Wang X, Coresh J, Greene T, Stevens L, et al. Cystatin $\mathrm{C}$ as a risk factor for outcomes in chronic kidney disease. Ann Intern Med. (2007) 147:19-27. doi: 10.7326/0003-4819-147-1-200707030-00004

9. Authors/Task Force m, Windecker S, Kolh P, Alfonso F, Collet JP, Cremer J, et al. 2014 ESC/EACTS guidelines on myocardial revascularization: the task force on myocardial revascularization of the European society of cardiology (ESC) and the European association for cardio-thoracic surgery (EACTS)developed with the special contribution of the European association of percutaneous cardiovascular interventions (EAPCI). Eur Heart J. (2014). 35:2541-619. doi: 10.1093/eurheartj/ehu278

10. Task Force M, Montalescot G, Sechtem U, Achenbach S, Andreotti F, Arden C, et al. 2013 ESC guidelines on the management of stable coronary artery disease: the task force on the management of stable coronary artery disease of the European society of cardiology. Eur Heart J. (2013) 34:29493003. doi: 10.1093/eurheartj/eht296

\section{FUNDING}

This study was supported by the National Key R\&D Program of China (2019YFA 0802300), the National Natural Science Foundation of China (81941005, 81970351, and 81822005), and the Clinical Research Award of the First Affiliated Hospital of XJTU (XJTU1 AF-CRF-2017-006).

\section{ACKNOWLEDGMENTS}

We thank Le zhao, Yaqiong Zhou and the Biobank of First Affiliated Hospital of Xi'an Jiaotong University for data collection.

\section{SUPPLEMENTARY MATERIAL}

The Supplementary Material for this article can be found online at: https://www.frontiersin.org/articles/10.3389/fcvm. 2020.586181/full\#supplementary-material

11. Stone GW, Kandzari DE, Mehran R, Colombo A, Schwartz RS, Bailey $S$, et al. Percutaneous recanalization of chronically occluded coronary arteries: a consensus document: part I. Circulation. (2005) 112:236472. doi: 10.1161/CIRCULATIONAHA.104.481283

12. Stone GW, Reifart NJ, Moussa I, Hoye A, Cox DA, Colombo A, et al. Percutaneous recanalization of chronically occluded coronary arteries: a consensus document: part II. Circulation. (2005) 112:25307. doi: 10.1161/CIRCULATIONAHA.105.583716

13. Ma YC, Zuo L, Chen JH, Luo Q, Yu XQ, Li Y, et al. Modified glomerular filtration rate estimating equation for Chinese patients with chronic kidney disease. J Am Soc Nephrol. (2006) 17:2937-44. doi: 10.1681/ASN.2006040368

14. Inker LA, Astor BC, Fox CH, Isakova T, Lash JP, Peralta CA, et al. KDOQI US commentary on the 2012 KDIGO clinical practice guideline for the evaluation and management of CKD. Am J Kidney Dis. (2014) 63:71335. doi: 10.1053/j.ajkd.2014.01.416

15. Campbell NG, Varagunam M, Sawhney V, Ahuja KR, Salahuddin N, De Palma R, et al. Mild chronic kidney disease is an independent predictor of long-term mortality after emergency angiography and primary percutaneous intervention in patients with ST-elevation myocardial infarction. Heart. (2012) 98:42-7. doi: 10.1136/heartjnl-2011-300024

16. Zhang QB, Chen LM, Li M, Cui YQ, Zhao CY, Cui LQ. Influence of chronic kidney disease on the outcome of patients with chronic total occlusion. Am J Transl Res. (2016) 8:196-208.

17. Budtz-Jorgensen E, Keiding N, Grandjean P, Weihe P. Confounder selection in environmental epidemiology: assessment of health effects of prenatal mercury exposure. Ann Epidemiol. (2007) 17:27-35. doi: 10.1016/j.annepidem.2006.05.007

18. Oterdoom LH, Gansevoort RT, Schouten JP, de Jong PE, Gans RO, Bakker SJ. Urinary creatinine excretion, an indirect measure of muscle mass, is an independent predictor of cardiovascular disease and mortality in the general population. Atherosclerosis. (2009) 207:534-40. doi: 10.1016/j.atherosclerosis.2009.05.010

19. Stevens LA, Schmid CH, Greene T, Li L, Beck GJ, Joffe MM, et al. Factors other than glomerular filtration rate affect serum cystatin C levels. Kidney Int. (2009) 75:652-60. doi: 10.1038/ki.2008.638

20. Shlipak MG, Katz R, Sarnak MJ, Fried LF, Newman AB, Stehman-Breen $\mathrm{C}$, et al. Cystatin $\mathrm{C}$ and prognosis for cardiovascular and kidney outcomes in elderly persons without chronic kidney disease. Ann Intern Med. (2006) 145:237-46. doi: 10.7326/0003-4819-145-4-200608150-00003

21. Peralta CA, Katz R, Sarnak MJ, Ix J, Fried LF, De Boer I, et al. Cystatin C identifies chronic kidney disease patients at higher risk for complications. $J$ Am Soc Nephrol. (2011) 22:147-55. doi: 10.1681/ASN.2010050483 
22. Flores-Blanco PJ, Lopez-Cuenca A, Januzzi JL, Marin F, Sanchez-Martinez M, Quintana-Giner $M$, et al. Comparison of risk prediction with the CKD-EPI and MDRD equations in non-ST-segment elevation acute coronary syndrome. Clin Cardiol. (2016) 39:507-15. doi: 10.1002/clc. 22556

23. Helmersson-Karlqvist J, Arnlov J, Larsson A. Cystatin C-based glomerular filtration rate associates more closely with mortality than creatininebased or combined glomerular filtration rate equations in unselected patients. Eur J Prev Cardiol. (2016) 23:1649-57. doi: 10.1177/204748731 6642086

24. Lim WH, Lewis JR, Wong G, Turner RM, Lim EM, Thompson PL, et al. Comparison of estimated glomerular filtration rate by the chronic kidney disease epidemiology collaboration (CKD-EPI) equations with and without Cystatin $\mathrm{C}$ for predicting clinical outcomes in elderly women. PLoS ONE. (2014) 9:e106734. doi: 10.1371/journal.pone. 0106734
25. Garcia-Carretero R, Vigil-Medina L, Barquero-Perez O, Goya-Esteban R, Mora-Jimenez I, Soguero-Ruiz C, et al. Cystatin C as a predictor of cardiovascular outcomes in a hypertensive population. J Hum Hypertens. (2017) 31:801-7. doi: 10.1038/jhh.2017.68

Conflict of Interest: The authors declare that the research was conducted in the absence of any commercial or financial relationships that could be construed as a potential conflict of interest.

Copyright (c) 2020 Li, Rong, Wang, Gao, Wen, Li, Cheng, Hua, Li, Jian, Zhang, Huang, Pan, Wu and Zhuo. This is an open-access article distributed under the terms of the Creative Commons Attribution License (CC BY). The use, distribution or reproduction in other forums is permitted, provided the original author(s) and the copyright owner(s) are credited and that the original publication in this journal is cited, in accordance with accepted academic practice. No use, distribution or reproduction is permitted which does not comply with these terms. 